Chronic Obstructive Pulmonary Diseases: Journal of the COPD Foundation

\author{
Editorial
}

\title{
Towards New Therapeutic Solutions for Alpha-1 Antitrypsin Deficiency: Role of the Alpha-1 Foundation
}

\author{
Adam Wanner, $\mathrm{MD}^{1}$
}

\author{
Abbreviations: alpha-1 antitrypsin deficiency, AATD; alpha-1 antitrypsin, AAT \\ Funding Support: not applicable \\ Citation: Wanner A. Towards new therapeutic solutions for alpha-1 antitrypsin deficiency: role of the Alpha-1 Foundation. Chronic Obstr \\ Pulm Dis. 2020;7(3):147-150. doi: https://doi.org/10.15326/jcopdf.7.3.2020.0141
}

\begin{abstract}
1 Division of Pulmonary, Allergy, Critical Care and Sleep Medicine, Department of Medicine, Miller School of Medicine, University of Miami, Miami, Florida
\end{abstract}

\section{Address correspondence to:}

\section{Adam Wanner, MD}

Phone: (305) 243-3045

Email: AWanner@med.miami.edu

\section{Keywords:}

alpha-1 antitrypsin deficiency; AATD; therapeutic solutions:

Alpha-1 Foundation

\section{Editorial}

Alpha-1 antitrypsin deficiency (AATD) is an autosomal co-dominant genetic condition that can result in chronic lung disease in adults and/or liver disease at any age. AATD occurs when the blood is deficient in a protein called alpha-1 antitrypsin (AAT). AAT is mainly produced by the liver, and one of its functions is to protect the lungs from increased protease activity, especially during episodes of inflammation caused by infection or inhaled irritants such as tobacco smoke. In most cases, AATD is caused by an abnormal folding of AAT. The misfolded AAT cannot be secreted from the liver at a normal rate, leading to a buildup of the abnormal AAT in the hepatocytes, which can cause liver disease, and a decrease of AAT in the blood, which can predispose to lung disease. Currently, only AAT augmentation therapy is available for the treatment of AATD-related lung disease. While the treatment can slow the progression of emphysema, it is not a cure for lung disease. ${ }^{1}$ At present, there are no specific therapeutic options for AATD-related liver disease.
Clearly, there is a need for new and better therapies.

Promoting research with the vision of finding better treatments and ultimately a cure for AATD is a core mission of the Alpha-1 Foundation. Investigatorinitiated grants have been the driving force of the Foundation's research program from its inception in 1999, based on the time-tested principle in biomedical discovery that innovative ideas typically arise in the research community. Over the past 2 decades, a significant portion of the program has been directed at the peer-reviewed, investigatorinitiated grants program. The program is based on the concept that investigator-initiated research leads to scientific publications and scientific meetings which in turn energize the investigators to remain engaged in alpha-1 research and inform biotech and pharmaceutical companies in their search for new therapeutic solutions (Figure 1).

Today we know the genetic mutations responsible for the misfolding of AAT, generally understand how the misfolded AAT polymerizes and leads to cellular processes that injure the liver and the lung (gain of function), and how the defect in AAT secretion into the blood circulation can expose the lung to the elastolytic actions of neutrophil elastase and other serine proteases leading to the development of chronic lung disease, mainly COPD (loss of function). Furthermore, we now have several in vitro and animal models to interrogate new therapies, know that AAT has broad anti-inflammatory and immunomodulatory actions that may benefit lung disease in patients with or without AATD, have demonstrated the rationale for gene-directed and cell-based therapies, and have 


\section{Figure 1. Alpha-1 Foundation Investigator-Initiated Grant Program}

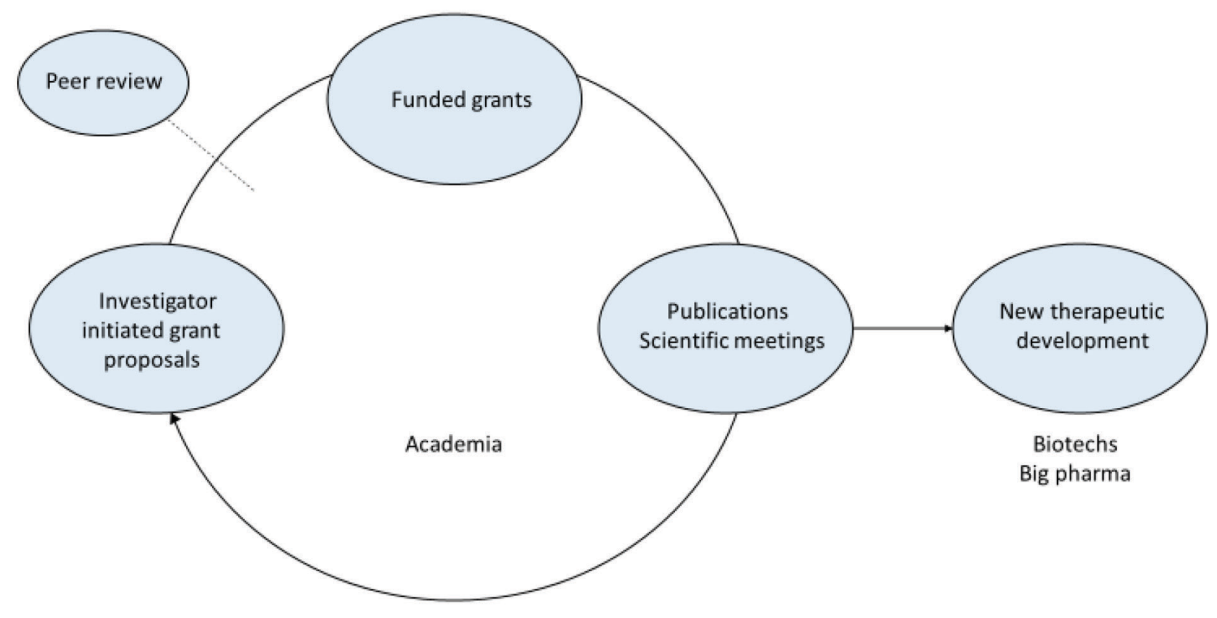

made some inroads into the identification of genetic and environmental modifiers that influence the clinical phenotype in AATD.

This research activity has identified new targets for therapeutic interventions with the potential of controlling or curing the liver and lung disease of AATD. It is likely that future therapeutic research will take 2 directions. One line of investigations will be directed at finding novel drugs, and gene-and cell-based interventions that target the basic defect in AATD; academia and biotech companies are best suited for this. The other area of therapeutic research is the development of new formulations of AAT for augmentation therapy and the search for new sources for AAT using recombinant or transgenic technology.

The proceedings of the 4th International Research Conference on Alpha-1 Antitrypsin, summarized in reviews in this issue of Chronic Obstructive Pulmonary Diseases: Journal of the COPD Foundation, reflect some of the progress made towards new diagnostic and therapeutic approaches for AATD-related lung and liver disease, based on a better understanding of the pathogenetic pathways leading to clinical disease. The conference was entitled, "Disease Progression and Therapeutic Options."

Concerning the pathogenesis of liver and lung disease, machine learning was proposed to understand DNA sequence to function to structure transformation in monogenic diseases such as AATD. While this "deep medicine" approach may bear fruit and identify new therapeutic targets in the future, there already exists a solid knowledge base to guide new therapeutic development. For example, it was reported that hepatic AAT polymer load correlates with hepatic fibrosis stage and long-term clinical outcome. With respect to lung disease, several speakers emphasized the role of inflammatory pathways not directly related to neutrophil elastase/AAT imbalance, the classical paradigm explaining the development of emphysema in AATD. AATD may be associated with an AATD neutrophil phenotype that amplifies neutrophilic inflammation, and enzymes other than serine proteases, pro-inflammatory cytokines and reactive oxygen species may be involved. Such investigations are fueled by the observation that AAT augmentation therapy slows but does not prevent the progression of emphysema. ${ }^{1}$

Another group of presentations addressed the clinical phenotype of AATD-related lung and liver disease. New non-invasive methods to detect and follow the progression of liver disease as well as genetic and environmental modifiers of liver and lung disease were discussed. With respect to specific therapy, only intravenous AAT augmentation for lung disease currently is available. The search for new treatments were reflected by talks that included inhaled AAT and anti-cytokines and antioxidants. While the material covered at the meeting was not allinclusive with respect to the current understanding of the mechanisms and potential therapeutic targets in AATD-related lung and liver disease, the information contained in the individual reviews/summaries should be of interest to the readership of this Journal.

Academic research has brought us to the verge of 
finding new treatments for AATD; however, the final step requires the help of the biotech and pharmaceutical industry that has the necessary resources to develop new therapies. Progress has been encouraging. In 2019, there were 15 AATD-directed clinical trials actively recruiting as listed on clinicaltrials.gov, including observational studies involving new formulations, applications and dosing of existing drugs, and most importantly, novel interventions targeting pathways that previously were identified by basic research to a great extent conducted in academic centers. It remains to be seen how many of these trials will lead to new Food and Drug Administration-approved treatments for AATD. However, the heightened clinical research activity demonstrates the indirect yet critical role of academic research in the quest for more effective therapeutic options in AATD. 


\section{References}

1. Chapman KR, Burdon JG, Piitlainen E, et al. Intravenous augmentation treatment and lung density in severe a1 antitrypsin deficiency (RAPID): a randomised, double-blind, placebo-controlled trial. Lancet. 2015;386(9991):360-368.

doi: https://doi.org/10.1016/s0140-6736(15)60860-1 